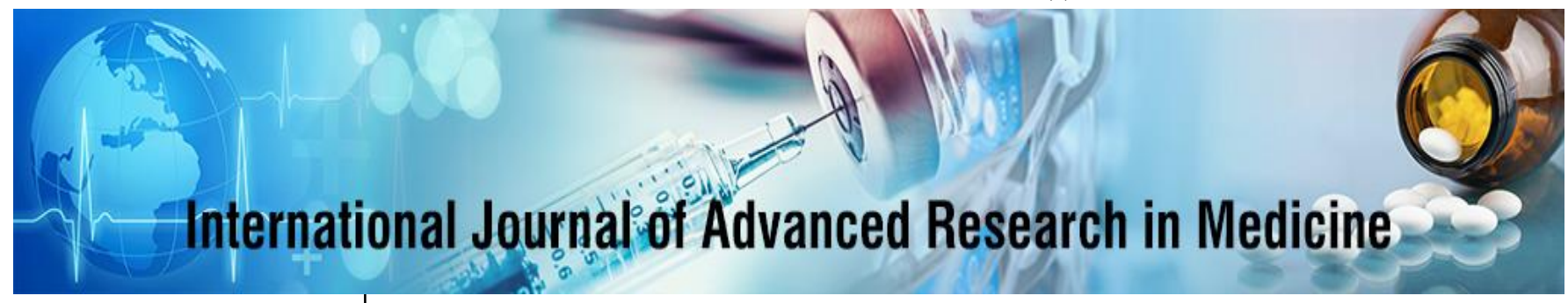

E-ISSN: 2706-9575

P-ISSN: 2706-9567

IJARM 2020; 2(2): 162-165

Received: 11-05-2020

Accepted: 21-06-2020

\section{Zainab Naji Hashim}

Ministry of Health, Baghdad Medical office, Al-Russafa,

Fatima Al-Zahraa Maternity Hospital for Women and

Children, Baghdad, Iraq

\section{Sana Abd Al Hadi Abed}

Ministry of Health, Baghdad Medical office, Al-Russafa

Fatima Al-Zahraa Maternity

Hospital for Women and

Children, Baghdad, Iraq

Rawaa Abdulraheem Hasan Ministry of Health, Baghdad Medical office, Al-Russafa, Fatima Al-Zahraa Maternity Hospital for Women and Children, Baghdad, Iraq
Corresponding Author: Zainab Naji Hashim Ministry of Health, Baghdad Medical office, Al-Russafa, Fatima Al-Zahraa Maternity Hospital for Women and Children, Baghdad, Iraq

\section{Treatment of anemia in the postpartum, postoperative period and immediate gynecological surgery with intravenous iron}

\author{
Zainab Naji Hashim, Sana Abd Al Hadi Abed and Rawaa Abdulraheem \\ Hasan
}

DOI: https://doi.org/10.22271/27069567.2020.v2.i2c.62

\begin{abstract}
Objective: To evaluate the efficacy and safety of intravenous iron infusion in the treatment of postpartum anemia and gynecological surgery.

Patients and methods: A prospective study conducted between June 2004 and December 2004, which included patients who were admitted to our resuscitation department and who had hemoglobin values below 10 after delivery or cesarean section and after gynecological surgery g.dL $\mathrm{dL}^{-1}$. Three $200 \mathrm{mg}$ doses of iron sucrose. Were administered intravenously on consecutive days. Fifteen days after the last dose, patients were called for analytical control and side effects were recorded. Use the student " $t$ " test to perform paired-sample analysis on the data.

Results: 257 obstetricians and 52 gynecologists received treatment, of which 156 and 33 patients were completed, respectively. After obstetric treatment, hemoglobin increased to $3.2 \mathrm{~g} . \mathrm{dL}^{-1}$, and in obstetric and gynecology patients it was 2.7 g.dL $\mathrm{dL}^{-1}$. Both results are statistically significant, $\mathrm{p}<0.001$, and the confidence interval is $95 \%$ (2.92-3.52 for obstetric cases and 2.22-3.07 for gynecological cases). Side effects were rare (13 obstetric patients and one gynecological patient) and the majority (12 of 14 women) had pain at the injection site.

Conclusion: Intravenous iron sucrose is safe and effective in the treatment of puerperal anemia and gynecological surgery. The low incidence of serious side effects and the rapid recovery of hemoglobin levels make it a safe and effective drug for the treatment of anemia.
\end{abstract}

Keywords: Anemia, Postpartum, Postoperative period, Iron deficiency

\section{Introduction}

Anemia is defined in non-pregnant women by hemoglobin ( $\mathrm{Hb})$ levels $<12 \mathrm{~g} \mathrm{dL}-1$, and in the case of pregnant women by hemoglobin $<11 \mathrm{~g} \mathrm{dL}-1$ in the first and third trimesters of gestation and $<10,5 \mathrm{~g} \mathrm{dL}-1$ in the second trimester ${ }^{[1]}$. Disorders during the physiological processes of labor and menstruation will be responsible for obstetric hemorrhage due to insufficient uterine involution, and chronic excessive blood loss during menstrual cycles due to an alteration in endometrial angiogenesis ${ }^{[2]}$, which makes these women prone patients to the need for transfusion ${ }^{[3,4]}$.

Iron deficiency anemia in pregnancy is an entity that appears in 10-30\% of pregnant women [5], and deserves special attention due to its potential consequences, both for the mother (greater susceptibility to contracting infections, lower numbers hemoglobin, decreased immune function) and the fetus (prematurity, delayed intrauterine growth, increased perinatal morbidity and mortality) ${ }^{[6]}$. Obstetricians recommend the administration of oral iron supplements during the second and third trimesters of gestation at a dose of $30 \mathrm{mg}$ of elemental iron per day ${ }^{[7]}$. The indications of the World Health Organization (WHO) are 60 $\mathrm{mg}$ a day in areas where the prevalence of iron deficiency is less than $20 \%$, and they advise doubling the dose when the prevalence is higher ${ }^{[7]}$. If situations of chronic bleeding during pregnancy are added to the anemia of pregnancy, such as the placenta previa, or bleeding greater than usual during childbirth or in the puerperal period, the risk of blood transfusion increases despite having fulfilled the indicated treatment with oral iron.

Anemia that appears in the postoperative period has, in addition to the active blood loss during the surgery, an inflammatory mechanism due to the surgery itself, which will lead to an alteration of iron metabolism (inhibited intestinal absorption) and the production and 
function of erythropoietin (suppression in the production of erythropoietin and the growth of erythroid colonies). This mechanism is mediated by the elevation of interleukin- 6 and C-reactive protein, and can last up to two weeks after surgery. During this period, oral iron administration is ineffective in correcting anemia. A similar inflammatory response has been observed in postpartum anemia ${ }^{[8-10]}$.

The clinical consequences of anemia include cardiovascular symptoms (hypotension, tachycardia), decreased physical and mental performance, fatigue and, in the case of women in the postpartum period, it represents a significant decrease in their ability to care for their newborn ${ }^{[11]}$. Anemia affects the activities of daily life, which translates into a deterioration in the quality of life ${ }^{[12]}$. Although today allogeneic transfusion is much safer than in the past in terms of the transmission of infectious diseases, it cannot be considered exempt from other

risks such as allergic, febrile and hemolytic reactions, transfusion errors, acute lung damage and immunomodulation disorders responsible for a higher incidence of postoperative infections and tumor recurrences [13-16]. In addition, the number of patients who refuse to be transfused is increasing. All of this is accompanied by a growing interest in alternative therapeutic options to blood transfusion, including the use of intravenous iron sucrose and recombinant human erythropoietin ${ }^{[17,18]}$.

In our Hospital we maintain a threshold of $7 \mathrm{~g} \mathrm{dL}-1$ of hemoglobin, always taking into account the associated diseases of each patient and tolerance to anemia, so that the decision transfusion is always individualized. Since June 2004, we have put into practice a protocol for the use of intravenous iron sucrose in all those patients, obstetric and gynecological, in which hemoglobin figures $<10 \mathrm{~g} \mathrm{dL}-1$ are observed during their postoperative stay in resuscitation, in order to assess the improvement of anemia after treatment and evaluate its safety through the appearance of side effects.

\section{Material and methods}

A prospective, open study was carried out from June to December 2004, which included all patients admitted to our Resuscitation Unit who, after delivery or in the immediate postoperative period of cesarean section or surgery Gynecologically, they presented anemia not susceptible to blood transfusion but which could hypothetically benefit from a faster recovery with intravenous iron therapy. Those women with hemoglobin values less than $10 \mathrm{~g} \mathrm{dL}-1$ were treated, dividing them for the study into two groups: obstetric and gynecological. All included patients gave their consent to receive the treatment.

The data collected were: age, iron profile and hemoglobin in the immediate postoperative period, that is, upon admission to resuscitation, type of surgery and, in the case of the obstetric group, type of delivery or cesarean section, together with the surgical indication for this last; adverse effects during the administration of the treatment (metallic taste, nausea, vomiting, headache, hypotension, pain at the injection site during the administration of the treatment, phlebitis, paraesthesia, fever, urticaria, skin rash, edema of the extremities, dyspnea, anaphylactoid reactions); need for transfusion during the treatment period or within 15 days; hemoglobin figures 15 days after completion of intravenous therapy.
Before starting treatment, a blood sample was drawn to obtain the iron profile, a study that includes levels of ferritin, transferrin, iron, iron transport capacity, and transferrin saturation index. The iron deficiency was calculated according to the formula: body weight $[\mathrm{kg}] \mathrm{x}$ (target $\mathrm{Hb}$-actual $\mathrm{Hb}$ ) [g L-1] x 0.24 + iron stores [mg]; where the desired hemoglobin $=150 \mathrm{~g} \mathrm{~L}-1$, and the iron stores $=500 \mathrm{mg}$ for patients weighing more than $35 \mathrm{~kg}$. Once the etiology of the anemia was found to be iron deficiency (the calculated iron deficiency exceeded $600 \mathrm{mg}$ in all patients), three doses of $200 \mathrm{mg} /$ day of intravenous iron sucrose were administered, on normal days. successive. The infusion was carried out diluted in $250 \mathrm{cc}$ of $0.9 \%$ physiological saline for one hour. The first dose was infused in Resuscitation, with the patient monitored with noninvasive blood pressure, electrocardiogram, and pulse oximetry. If no adverse effects appeared during the first dose, the plant treatment was completed the next two days. The total dose administered was $600 \mathrm{mg}$, except in cases in which side effects appeared. The reason for performing the treatment on three consecutive days was to take advantage of the hospital stay, since obstetric patients only remain hospitalized for three days.

The exclusion criteria were: allergy to iron; non-iron deficiency anemia, hemolytic or thalassemia; hemochromatosis and hemosiderosis; ferritin> $200 \mathrm{ng} \mathrm{dL}-1$; hematological disease; liver function disorder (bilirubin $>2$ g dL-1, and liver enzymes - aspartate aminotransferase or GOT and alanine aminotransferase or GPT- greater than twice the baseline value); positive serology for hepatitis B, $\mathrm{C}$ or human immunodeficiency virus; acute or chronic infection; severe asthma and atopy; need for transfusion during delivery, surgery, or during the study period; and the non-consent of the patient. These exclusion criteria were adopted taking into account the contraindications and precautions of the drug's technical data sheet. The value of $200 \mathrm{ng}$ dL-1 for ferritin was taken to be the upper limit of normality given by our laboratory: since it indicates the value of the organism's iron deposits, above this figure, With treatment, we could lead to iron overload. Positive serology for hepatitis viruses was added to rule out possible unknown liver dysfunction. The transfusion of packed red blood cells is not a contraindication for treatment with intravenous iron, but since $200 \mathrm{mg}$ of iron is administered with each packed red blood cells, if we performed the intravenous treatment simultaneously we could cause an overload of the iron transport system and cause toxicity.

Fifteen days after the last dose, the patients were summoned for an analytical control with a hemogram, being a sine qua non condition for completing the study not to take oral iron during this time. After receiving the results, the patients were informed of the results by telephone, indicating the need or not to continue oral iron therapy.

The data were processed by computer using a database in Microsoft Excel format, which was later imported for statistical treatment in the SPSS version 11 program. For the description of continuous quantitative variables, the mean was used together with the deviation standard. The qualitative variables were described using absolute frequencies and relative frequencies expressed as a percentage. In the comparison of quantitative data between two groups, the parametric Student's t test was used. Differences were considered significant at $\mathrm{p}<0.001$. 


\section{Results \\ Obstetric patients}

257 women were included in this group. 230 underwent cesarean section, 14 underwent forceps, and 13 had a normal vaginal delivery. Of the 230 caesarean sections, 75 were indicated by non-progression of labor or failure of induction; 49 were due to breech presentation, transverse situation or maternal pathology; 48 due to previous caesarean section; 33 for risk of loss of fetal well-being; 13 were cesarean sections in patients with a history of hemorrhagic risk (placenta previa); 11 were cesarean section in multiple gestations.

Of the total number of women seen, 245 received the three doses of treatment. Only 156 of the total $(60.7 \%)$ completed the study, since 79 of them $(30.7 \%)$ did not show up for analytical control fifteen days after treatment; in $11(4.3 \%)$ there were errors in filling in the forms given to the patients and another $11(4.3 \%)$ did not complete the treatment for other reasons: 8 were administered oral iron in the hospital ward despite having started intravenous therapy and the remaining three required blood transfusion due to puerperal hemorrhage.

The data obtained from the obstetric patients treated. The hemoglobin values before starting the treatment ranged between $6.8 \mathrm{~g} \mathrm{dL}-1$ the minimum and $9.9 \mathrm{~g} \mathrm{dL}-1$ the maximum. The mean increase in the hemoglobin level in the obstetric group, fifteen days after treatment with intravenous iron sucrose, was $3.2 \mathrm{~g} \mathrm{dL}-1$ ( $\mathrm{p}<0.001 ; 95 \%$ confidence interval: 2.92- 3.52).

Regarding adverse reactions, 13 patients $(5 \%)$ suffered side effects, 12 of them related to the presence of pain in the infusion area, one of the latter being complicated by the appearance of an abscess in the arm when extravasating the medication during the last dose. It resolved after 10 days of local treatment with pentosan polysulfate sodium and oral cloxacillin $500 \mathrm{mg}$ every 6 hours, without the need for debridement. Patient thirteen began with papular, erythematous, and pruritic lesions 10 minutes after starting the infusion. This patient suffered from chronic atopic dermatitis that had not been reflected in the medical history. The skin reaction disappeared with the immediate cessation of the infusion and by intravenous treatment with $5 \mathrm{mg}$ of dexchlorpheniramine maleate and $100 \mathrm{mg}$ of hydrocortisone sodium phosphate. Of the patients who presented adverse effects, the latter was the only one to whom treatment was suspended after the clinical picture that appeared during the administration of the first dose, and was excluded from the study.

\section{Gynecological patients}

52 women who underwent gynecological surgery were treated. The largest number of patients corresponded to abdominal hysterectomies indicated for uterine myxomatosis (22 patients, $42.3 \%$ of the total) followed by those patients who underwent myomectomy (11 women, $21.2 \%) ; 8(15.4 \%)$ underwent breast surgery (mastectomy with axillary lymphadenectomy or bilateral mastectomy with reconstruction in the same surgical act); 5 patients $(9.6 \%)$ required treatment

treatment after undergoing emergency surgery for ectopic pregnancy with hemoperitoneum; 2 after being operated on for a ruptured ovarian cyst; 2 after surgery for ovarian cancer and 2 in vaginal hysterectomies for uterine prolapse. Of the 52 patients who started the study, only 33 completed it. Of the remaining 19, 13 did not carry out the analytical control at fifteen days, in four cases the data were collected incompletely and in another two cases were administered oral iron in the hospital ward after having started the intravenous treatment. These last two patients were the only ones who did not complete treatment.

The data obtained from these patients. The mean increase in the hemoglobin level in the patients of the gynecological group, 15 days after treatment with intravenous iron sucrose, was $2.7 \mathrm{~g} \mathrm{dL}-1$ ( $\mathrm{p}<0.001 ; 95 \%$ confidence interval: 2.20-3.07).

During treatment, only one patient $(1.9 \%)$ had pain in the injection site as an adverse effect.

\section{Discussion}

In the postpartum period, $30 \%$ of women will be affected by anemia with hemoglobin levels $<10 \mathrm{~g} \mathrm{dL}-1$ and up to $10 \%$ will suffer severe anemia (hemoglobin $<8$ g dL-1) 19. Added to the ineffectiveness of oral iron treatment in cases of severe anemia is the high incidence of non-compliance due to the appearance of side effects (10-40\% of adverse effects, mainly gastrointestinal) 8 . The safety of the new intravenous iron preparations is also not comparable with those available up to now ${ }^{[20-23]}$.

The largest number of patients in the obstetric group was represented by those women who underwent cesarean section due to failure of induction or non-progression of labor $(29.3 \%$ of the total), which is within the expectation since they are fulfilled. At least two of the risk factors for the appearance of obstetric hemorrhage: prolonged labor and stimulation with oxytocin ${ }^{[24,25]}$. If we consider a history of previous cesarean section as a risk factor for increased bleeding during the following surgery and we add it to the group of patients who had some other history of bleeding risk (placenta previa, total occlusive placenta, anterior uterine surgery), they make up the second most frequent obstetric group (23.8\%). In the case of multiple pregnancies, a more pronounced drop in hemoglobin levels has been described, which appears to be physiological, in relation to single pregnancies ${ }^{[26]}$, so it is not surprising that in our sample there are patients with multiple pregnancies.

Women with vaginal deliveries are not routinely admitted to the Resuscitation Unit unless there is more bleeding than usual ${ }^{[25,27,28]}$. The fact of having treated some patients after vaginal, normal or instrumented delivery only serves as descriptive data for the sample of patients treated. Some of these patients are treated by the obstetrician, with the same protocol described, on the hospital ward, although the latter are not included in the present study.

In the group of patients included after gynecological surgery, as expected, the largest number of women corresponded to surgeries indicated for uterine myomatosis4: $42.3 \%$ hysterectomies and $21.2 \%$ myomectomies.

\section{References}

1. Centers for Disease Control (CDC) criteria for anemia in children and childbearing-aged women. MMWR Morb Mortal Wkly Rep 1989;38(22):400-404.

2. Boyd ME. Transfusion alternatives in Obstetrics / Gynecology. In: Transfusion Medicine and Alternatives to Blood Transfusion. Network for Advancement of Transfusion Alternatives. R\&J Editions Medicales. Chapter VII, 468-480. 
3. Stehling L. Gender-related variation in transfusion practices. Transfusion 1997;38:392-399.

4. Santoso JT, Lin DW, Miller DS. Transfusion medicine in Obstetrics and Gynecology. Obstet Gynecol Surv 1995;50(6):470-481.

5. Carson JL, Ferreira G. Transfusion triggers: how low can we go? Vox Sanguinis 2004;87:S218-S221.

6. Scholl TO, Hediger ML, Fisher RL, Sheaver JW. Anemia versus iron deficiency: increased risk of preterm delivery in a prospective study. Am J Clin Nutr 1992;55(5):985-988.

7. Bashiri A, Burstein E, Sheiner E, Mazor M. Anemia during pregnancy and treatment with intravenous iron: review of the literature. Eur J Obstet Gynecol Reprod Biol 2003;110(1):2-7.

8. Biesma DH, Van de Wiel A, Begin Y, Kraaijenhagen RJ, Marx JIM. Postoperative erythropoiesis is limited by the inflammatory effect of surgery on iron metabolism. Eur J Clin Invest 1995;25(11):383-389.

9. Breymann C, Richter C, Huttner C, Huch R, Huch A. Effectiveness of recombinant erythropoietin and iron sucrose vs. iron therapy only, in patients with postpartum anaemia and blunted erythropoiesis. Eur J Clin Invest 2000;30(2):154-161.

10. Beris P. The use of iron to increase red cell mass. Can J Anesth 2003;50(6 Suppl):S3-S9.

11. Bruner AB, Joffe A, Duggan AK, Casella JF, Brandt J. Randomized study of cognitive effects of iron supplementation in nonanaemic iron- deficient adolescent girls. Lancet 1996;348(9033):992-996.

12. Carson JL, Terrin ML, Jay M. Anemia and postoperative rehabilitation. Can J Anesth 2003;50(6 Suppl):S60-S64.

13. Hill GE, Frawley WH, Griffith KE, Forestner JE, Minei JP. Allogeneic blood transfusion increases the risk of postoperative bacterial infection: a meta-analysis. J Trauma 2003;54(5):908-914.

14. Heiss MM. Risk of allogeneic transfusions. $\mathrm{Br} \mathrm{J}$ Anaesth 1998;81(Suppl 1):16-19.

15. Ortega LF, Muerza JE. Anaphylactic transfusion reaction, under general anesthesia, caused by anti-IgA antibodies. Rev Esp Anestesiol Reanim 1985;32(1):3940.

16. López-Andrade Jurado A, Almazán Duro A, Martín Ruiz JL, Samanie- go Muñoz F, López-Andrade Jurado MA, Del Campo Iglesias A. Immune response in the surgical patient: effect of anesthesia and blood transfusion. Rev Esp Anestesiol Reanim 2000;47(2):6780.

17. Breymann C. Iron deficiency and anemia in pregnancy: modern aspects of diagnosis and therapy. Blood Cells Mol Dis 2002;29(3):506-516.

18. Vora M, Gruslin A. Erythropoietin in obstetrics. Obstet Gynecol Surv 1998;53:500-508.

19. Ekeroma A, Ansari A, Stirrat G. Blood transfusions in obstetrics and gynaecology. Br J Obstet Gynaecol 1997;104(3):278-284.

20. Faich G, Strobos J. Sodium ferric gluconate complex in sucrose: safer intravenous iron therapy than iron dextrans. Am J Kidney Dis 1999;33(3):464-470.

21. Geiser P, Baer M, Schaub E. Structure / hitotoxicity relationship of parenteral iron preparations. Drug Res 1992;42(12):1439-1452.

22. Hamstra RD, Block MH, Schoket AL. Intravenous iron dextran in clinical medicine. JAMA 1980;243(17):1726-1731.

23. Fahmy K. Systemic reactions with total dose infusion of iron dextran complex in obstetric patients. Int $\mathrm{J}$ Gynaecol Obstet 1978-79;16(2):170-172.

24. Magann EF, Evans S, Chauhan SP, Lanneau G, Fisk $\mathrm{AD}$, Morrison JC. The length of the third stage of labor and the risk of postpartum hemorrhage. Obstet Gynecol 2005;105(2):290-293.

25. Download G, Pirette P, Gravier A, Roman H, Lemoine JP, Marpeau L. Missed diagnosis of postpartum hemorraghe. J Gynecol Obstet Biol Reprod 2001;30(6):590-600.

26. Krafft A, Breymann C, Streich J, Huch R, Huch A. Hemoglobin concentration in multiple versus singleton pregnancies. Retrospective evidence for physiology not pathology. Eur J Obstet Gynecol Reprod Biol 2001;99(2):184-187.

27. Petersen LA, Lindner DS, Kleiber CM, Zimmerman MB, Hinton AT, Yankowitz J. Factors that predict low hematocrit levels in the postpartum patient after vaginal delivery. Am J Obstet Gynecol 2002;186(4):737-744.

28. Swaim LS, Perriatt S, Andres RL, Paradissis J, Watson MN. Clinical utility of routine postpartum hemoglobin determinations. Am J Perina- tol 1999;16(7):333-337.

29. Bayoumeu F, Subiran-Buisset C, Baka NE, Legagneur $\mathrm{H}$, Monnier- Barbarino P, Laxenaire MC. Iron therapy in iron deficiency anemia in pregnancy: intravenous route versus oral route. Am $\mathbf{J}$ Obstet Gynecol 2002;186(3):518-522.

30. Brooch DE, Gay C, Armand-Branger S, Granjease L, Terzibachian JJ. Acute postpartum anaemia. Clinical practice and interest of intravenous iron. Gynecol Obstet Fertil 2004;32(7-8):613-619.

31. Perewusnyk G, Huch R, Huch A, Breymann C. Parenteral iron therapy in obstetrics: 8 years experience with iron-sucrose complex. Br J Nutr 2002;88(1):3-10.

32. Carmel R. Anemia and aging: an overview of clinical, diagnostic and biological issues. Blood Rev 2001;15(1):9-18.

33. Cuenca J, García Erce JA, Muñoz M, Izuel M, Martínez AA, Herrera A. Patients with pertrochanteric hip fracture may benefit from preoperative intravenous iron therapy: a pilot study. Transfusion 2004;44(10):14471452.

34. Al-Momen AK, Al-Meshari A, Al Nuaim L, Saddique A, Abotalib Z, Khashogji $\mathrm{T}$, et al. Intravenous iron sucrose complex in the treatment of iron deficiency anemia during pregnancy. Eur J Obstet Gynecol Reprod Biol 1996;69(2):121-124. 